\title{
DATA SCIENCE: IS IT STATISTICS OR COMPUTER SCIENCE? STATISTICS EDUCATION IN THE AGE OF BIG DATA
}

Timothy J. Kyng, Ayse Bilgin and Busayasachee Puang-Ngern Macquarie University, Australia

Timothy.Kyng@mq.edu.au

Big Data / Data Science is a very important emerging area for statisticians. Software skills are increasingly important for statistical practitioners. Data science may be regarded by statisticians as a new name for statistical science but in industry and government the perception may be different. Recent advances in IT have enabled us to collect, store and easily access large amounts of data with modest cost. The capacity to analyse the data and use it for decision making has lagged behind. Software has been developed to filter, access and analyse data. Computer scientists and statisticians have been working separately, not jointly on this. This paper explores the implications of Big Data for statisticians' education and aims to identify what skills are needed and software packages to use as well as the gaps between the perceptions of practitioners and academics about these issues. 